\title{
Effects of planetary mass accretion on the planets migration: the disk structures
}

\author{
Ramiro Álvarez and Erick Nagel \\ Departamento de Astronomía, Universidad de Guanajuato, \\ Guanajuato, Gto 36240, Mexico \\ email: ramiro@astro.ugto.mx
}

\begin{abstract}
We develop numerical tools to detect and quantify the mass and area for all structures of a protoplanetary disk with different optical depths. We consider two planets of $0.1 \mathrm{M}_{\mathrm{J}}$. They are initially at 12.5 and $20 \mathrm{AU}$ embedded in a viscous disk, where planetary mass accretion and migration are allowed. We find a gap region with optically thick streams, this region is characterized by an optically thin filling factor near to 1 . Additionally, we generate the Spectral Energy Distribution of each structure of the disk considering silicate dust. Our tools are useful to identify the locations of structures and sub-structures as possible sites where the material is accumulated.
\end{abstract}

Keywords. planets and satellites: formation, planetary systems: protoplanetary disk, structure formation, pre-transitional disk, transitional disk

\section{Introduction}

The transitional disks show optically thick emission of hot dust close to the star (Calvet et al. 2005), while pre-transitional disks show optically thin emission (Espaillat et al. 2007). They have (1) mass accretion rate onto the star of $\sim 10^{-10}-10^{-8} \mathrm{M}_{\odot} / \mathrm{yr},(2)$ regions with holes or cavities with sizes around tens of AU. Despite the lack of dust, the observed stellar accretion rates are inconsistent to dust-to-gas standard ratio.

A feasible way to explain this kind of disks is the planetary mass accretion. This problem was previously studied by Zhu et al. (2011), They considered accretion rates of mass onto the planets, which first are able to form a gap and then an internal hole. Furthermore, Dodson-Robinson \& Salyk (2011) suggested that the planetary system induces a gap and structures formation into the hole. These structures consist of currents (like filaments) and an optically thick residual inner disk, and they can be optically thick or optically thin. Particularly, the optically thick region can hide disk material.

In this work, we perform hydrodynamical simulations with FARGO code (Masset 2000). Additionally, we develop numerical tools to quantify the physical properties of disk structures. Finally, considering silicate dust, we make the SEDs of each disk structure using RADMC3D (Dullemond et al. in prep).

\section{Initial conditions}

For the simulations we use the initial conditions similar to Zhu et al. (2011): 1) the disk density is $\Sigma=178(r / \mathrm{AU})^{-1}(0.01 / \alpha) \mathrm{g} \mathrm{cm}^{-2}$, with $\alpha=0.1$, (Shakura \& Sunyaev $1973)$, in steady state with accretion stellar rate of $10^{-8} \mathrm{M}_{\odot}$, the central stellar mass is $\left.1 \mathrm{M}_{\odot} ; 2\right)$ the disk has $0.75-200 \mathrm{AU}$ in size, resulting in $0.025 \mathrm{M}_{\odot} ; 3$ ) the temperature radial profile (isothermal vertically) is $\left.T=221(r / \mathrm{AU})^{-1 / 2} \mathrm{~K} ; 4\right)$ the disk aspect ratio is $h / r=0.029(r / \mathrm{AU})^{0.25}$, where $h$ is the height scale; 5$)$ the planetary accretion is 

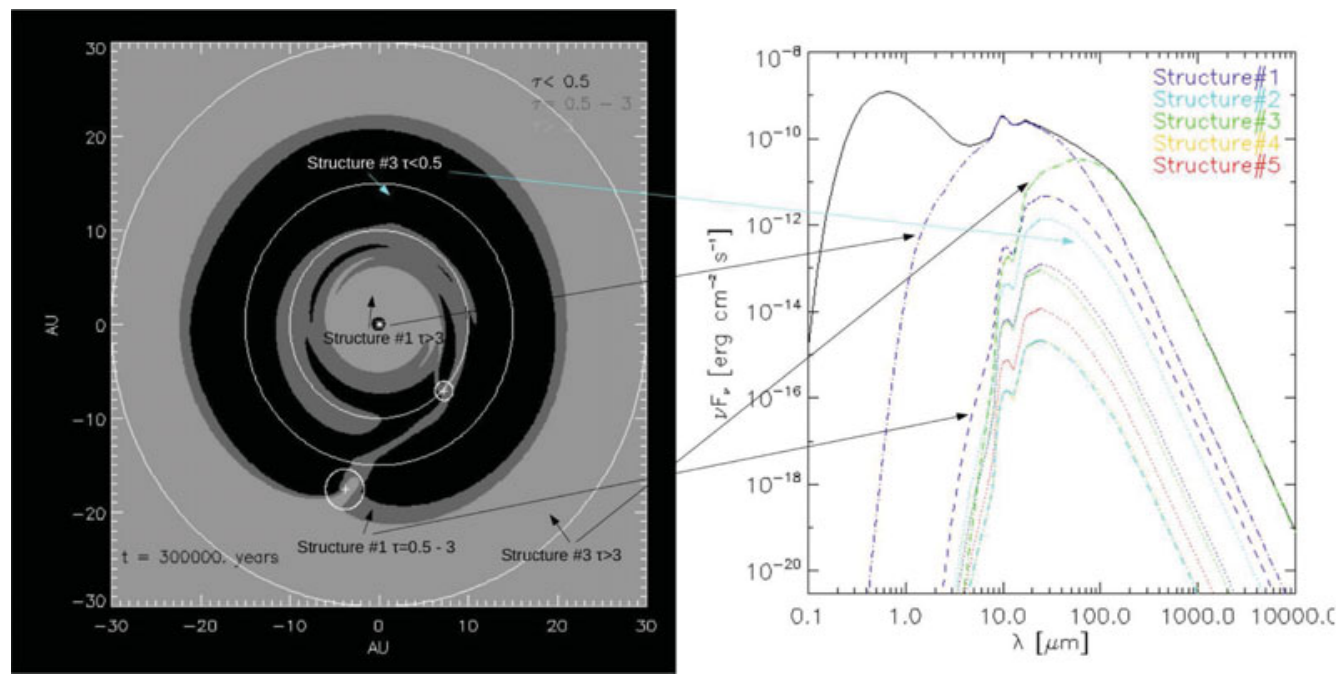

Figure 1. Snapshots at $3 \times 10^{5}$ years. Connection between the structures on ranges of optical depths and their SEDs. Left: Great circles shows radii at 10, 15 and 30 AU. The small ones shows the Hill Radii of the planets. The number of structure with their respective range on optical depth is showed by arrows. Right: SEDs at 140 pc. The black line shows the total emission. The colors (see electronic version) correspond to individual structure. The lines: doted, dashed and dash dot, correspond to structures optically thin, intermediate and thick, respectively.

parametrized by the $\alpha$ parameter inside of the Hill Radii of the planets $\alpha_{\mathrm{p}}=0.4$, the case showed here only consider initially two planets with $0.1 \mathrm{M}_{\mathrm{J}}$.

\section{Results and conclusions}

In order to characterize the structures, we consider the following physical characteristics of the dust: 1) ratio 1:100 of dust-to-gas; 2) Silicates, Organics and Troilite with $R_{\min }=0.005 \mu \mathrm{m}, R_{\max }=0.25 \mu \mathrm{m}$, size distribution of $R^{-3.5}$, and opacity at $10 \mu \mathrm{m}$ of $9.6 \mathrm{~cm}^{2} \mathrm{~g}^{-1}$. We use iteration of the optical depth, as a method to find structures on different opacity ranges. This means that neighbour cells with the same range of optical depth become a member of the same structure.

The figure 1 (left), shows a snapshot at $3 \times 10^{5}$ years with structures and ranges on optically depth: $\tau<0.5$ (thin), $0.5<\tau<3$ (intermediate), $3<\tau$ (thick). Every found structure has a corresponding SED in figure 1 (Right).

The tools shown here allow us to constrain all the disk by structures or substructures, and to explore physical quantities such as the mass, area, temperature and spectrum. Specially, the region of the spiral arm between planets is well characterized.

\section{References}

Calvet, N., D’Alessio, P., Watson, D. M., Franco-Hernández, R., Furlan, E., Green, J., Sutter, P. M., Forrest, W. J., Hartmann, L., Uchida, K. I., Keller, L. D., Sargent, B., Najita, J., Herter, T. L., Barry, D. J., \& Hall, P. 2005, ApJ, 630, 185

Dodson-Robinson, S. E. \& Salyk, C. 2011, ApJ, 738, 131

Espaillat, C., Dalvet, N., D’Alessio, P., Hernández, J., Qi, C., Hartmann, L., Furlan, E., \& Watson, D. M. 2007, ApJ, 670, 135

Masset, F. 2000, A\& $A, 141,165$

Zhu, Z., Nelson, R. P., Hartmann, L., Espaillat, C., \& Calvet, N. 2011, ApJ, 729, 47 